\title{
Particle Size is a Primary Determinant for Sigmoidal Kinetics of Nanoparticle Formation: A “disproof” of the Finkey-Watzky (F-W) nanoparticle nucleation and growth mechanism.
}

\author{
James D. Martin \\ Department of Chemistry, North Carolina State University, Raleigh, NC 27695-8204
}

Supporting Information

Simulated Data 
Restricted growth of a Sphere $\left(V=4 / 3 \pi\left(k_{g} t\right)^{3}\right)$ Within a Unit Cube

\begin{tabular}{|c|c|c|}
\hline Time & $\begin{array}{l}\text { Volume } \\
k_{\mathrm{g}}=0.02\end{array}$ & $\begin{array}{l}\text { Volume } \\
\mathrm{k}_{\mathrm{g}}=0.04\end{array}$ \\
\hline 0 & 0.00000000 & 0.00000000 \\
\hline 1 & 0.00003351 & 0.00026808 \\
\hline 2 & 0.00026808 & 0.00214466 \\
\hline 3 & 0.00090478 & 0.00723823 \\
\hline 4 & 0.00214466 & 0.01715728 \\
\hline 5 & 0.00418879 & 0.03351032 \\
\hline 6 & 0.00723823 & 0.05790584 \\
\hline 7 & 0.01149404 & 0.09195232 \\
\hline 8 & 0.01715728 & 0.13725828 \\
\hline 9 & 0.02442902 & 0.19543220 \\
\hline 10 & 0.03351032 & 0.26808257 \\
\hline 11 & 0.04460224 & 0.35681790 \\
\hline 12 & 0.05790584 & 0.46324669 \\
\hline 13 & 0.07362218 & 0.58510697 \\
\hline 14 & 0.09195232 & 97504 \\
\hline 15 & 0.11309734 & 6453 \\
\hline 16 & 0.13725828 & 0.87885845 \\
\hline 17 & 0.16463621 & 0.93843980 \\
\hline 18 & 0.19543220 & 0.9829228 \\
\hline 19 & 0.22984730 & 0.9939105 \\
\hline 20 & 0.26808257 & 0.99876455 \\
\hline 21 & 33909 & \\
\hline 22 & 0.35681790 & 0000 \\
\hline 23 & 0.40772008 & 000 \\
\hline 24 & 0.46324669 & 1.00000000 \\
\hline 25 & 0.52359878 & 1.00000000 \\
\hline 26 & 0.58510697 & 1.00000000 \\
\hline 27 & 0.64369977 & 1.00000000 \\
\hline 28 & 504 & 0000 \\
\hline 29 & 0.7 & 0000 \\
\hline 30 & 0.7 & 0000 \\
\hline 31 & 0.84087450 & 1.00000000 \\
\hline 32 & 0.87885845 & 1.00000000 \\
\hline 33 & 0.91151426 & 1.00000000 \\
\hline 34 & 0.93843980 & 1.00000000 \\
\hline 35 & 0.95923296 & 00000 \\
\hline 36 & 0.97524879 & 1.00000000 \\
\hline 37 & & 1.00000000 \\
\hline 38 & 0.9 & 00000 \\
\hline 39 & 0.99414899 & 1.00000000 \\
\hline 40 & 0.99691660 & 1.00000000 \\
\hline 41 & 0.99834206 & 1.00000000 \\
\hline 42 & 0.99882333 & 1.00000000 \\
\hline 43 & 0.99885048 & 1.00000000 \\
\hline 44 & 1.0 & 0000 \\
\hline 45 & 1.00000000 & 1.00000000 \\
\hline 46 & 1.00000000 & 1.00000000 \\
\hline 47 & 1.00000000 & 1.00000000 \\
\hline 48 & 1.00000000 & 1.00000000 \\
\hline 49 & 1.00000000 & 1.00000000 \\
\hline 0 & & \\
\hline
\end{tabular}

\section{Unrestricted Growth}

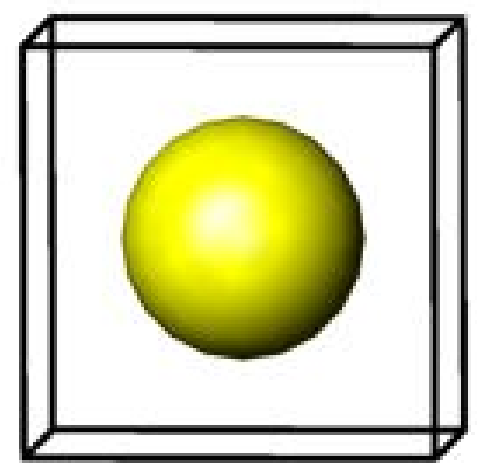

Face Impingement

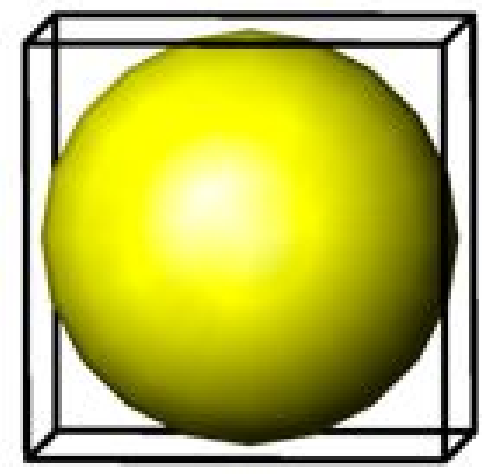

Face + Edge Impingement

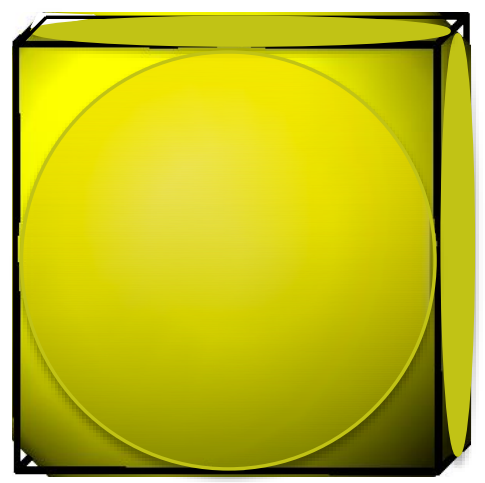


Spherical Growth $\left(V=4 / 3 \pi\left(k_{g} t\right)^{3}\right), k_{g}=0.02$, With Varying Nucleation Rates

\begin{tabular}{|c|c|c|c|c|}
\hline $\begin{array}{l}\text { Nuc } \\
\text { Rate }\end{array}$ & Single & $\begin{array}{l}5,1 \text { per } 3 \\
\text { time steps }\end{array}$ & $\begin{array}{l}5,1 \text { per } \\
\text { time step }\end{array}$ & 5 burst \\
\hline Time & Volume & Volume & Volume & Volume \\
\hline 0 & 0.00000000 & 0.00000000 & 0.00000000 & 0.00000000 \\
\hline 1 & 0.00003351 & 0.00003351 & 0.00003351 & 0.00016755 \\
\hline 2 & 0.00026808 & 0.00026808 & 0.00030159 & 0.00134041 \\
\hline 3 & 0.00090478 & 0.00090478 & 0.00120637 & 0.00452389 \\
\hline 4 & 0.00214466 & 0.00217817 & 0.00335103 & 0.01072330 \\
\hline 5 & 0.00418879 & 0.00445687 & 0.00753982 & 0.02094395 \\
\hline 6 & 0.00723823 & 0.00814301 & 0.01474454 & 0.03619115 \\
\hline 7 & 0.01149404 & 0.01367221 & 0.02597050 & 0.05747020 \\
\hline 8 & 0.01715728 & 0.02161416 & 0.04222301 & 0.08578642 \\
\hline 9 & 0.02442902 & 0.03257203 & 0.06450737 & 0.12214512 \\
\hline 10 & 0.03351032 & 0.04718253 & 0.09382890 & 0.16755161 \\
\hline 11 & 0.04460224 & 0.06621640 & 0.13119291 & 0.22301119 \\
\hline 12 & 0.05790584 & 0.09047787 & 0.17760470 & 0.28952918 \\
\hline 13 & 0.07362218 & 0.12080471 & 0.23406960 & 0.36811088 \\
\hline 14 & 0.09195232 & 0.15816872 & 0.30159289 & 0.45976161 \\
\hline 15 & 0.11309734 & 0.20357520 & 0.38117991 & 0.56548668 \\
\hline 16 & 0.13725828 & 0.25802948 & 0.47383595 & 0.68629139 \\
\hline 17 & 621 & 3685 & 56632 & 8105 \\
\hline 18 & 220 & 262 & 37634 & 098 \\
\hline 19 & 0.22984730 & 0.48573211 & 0.84027132 & 1.00000000 \\
\hline 20 & 0.26808257 & 0.58643063 & 0.99525655 & 1.00000000 \\
\hline 21 & 0.31033909 & 0.70120348 & 1.00000000 & 1.00000000 \\
\hline 22 & 0.35681790 & 0.83105598 & 1.00000000 & 1.00000000 \\
\hline 23 & 0.40772008 & 0.97699343 & 1.00000000 & 1.00000000 \\
\hline 24 & 0.46324669 & 00000 & 000000 & 00000 \\
\hline 25 & 878 & 000 & 0000 & 0000 \\
\hline 26 & 0.58897741 & 0000 & 1.00000000 & 00000 \\
\hline 27 & 0.65958366 & 1.00000000 & 1.00000000 & 00000 \\
\hline 28 & 0.73561858 & 1.00000000 & 1.00000000 & 1.00000000 \\
\hline 29 & 0.81728323 & 1.00000000 & 1.00000000 & 1.00000000 \\
\hline 30 & 0.90477868 & 1.00000000 & 1.00000000 & 1.00000000 \\
\hline 31 & 0.99830599 & 1.00000000 & 1.00000000 & 1.00000000 \\
\hline 32 & & 1.0 & 000 & 0000 \\
\hline 33 & 00 & 000 & 000 & 000 \\
\hline 34 & 1.00000000 & 1.00000000 & 1.00000000 & 1.00000000 \\
\hline 35 & 1.00000000 & 1.00000000 & 1.00000000 & 1.00000000 \\
\hline 36 & 1.00000000 & 1.00000000 & 1.00000000 & 1.00000000 \\
\hline 37 & 1.00000000 & 1.00000000 & 1.00000000 & 1.00000000 \\
\hline 38 & 1.00000000 & 1.00000000 & 1.00000000 & 1.00000000 \\
\hline 39 & & 1.00000000 & 1.00000000 & 1.00000000 \\
\hline 40 & & 1.0 & 00 & 000 \\
\hline 41 & 1.00000000 & 1.00 & 00000 & 1.00000000 \\
\hline 42 & 1.00000000 & 1.00000000 & 1.00000000 & 1.00000000 \\
\hline 43 & 1.00000000 & 1.00000000 & 1.00000000 & 1.00000000 \\
\hline 44 & 1.00000000 & 1.00000000 & 1.00000000 & 1.00000000 \\
\hline 45 & 1.00000000 & 1.00000000 & 1.00000000 & 1.00000000 \\
\hline 46 & 1.00000000 & 1.00000000 & 1.00000000 & 1.00000000 \\
\hline 47 & 1.00000000 & 1.00000000 & 1.00000000 & 1.00000000 \\
\hline 48 & 1.00000000 & 1.00000000 & 1.00000000 & 1.00000000 \\
\hline 49 & & 1.00000000 & 1.00000000 & 1.00000000 \\
\hline 50 & 1.00000000 & 1.00000000 & 1.00000000 & 1.00000000 \\
\hline
\end{tabular}


Spherical Growth $\left(V=4 / 3 \pi\left(k_{g} t\right)^{3}\right), k_{g}=0.02,1$ Nuc. Per Time Step, With Varying Induction Times

\begin{tabular}{|c|c|c|c|c|}
\hline $\begin{array}{l}\text { Induct } \\
\text { time }\end{array}$ & 0 & 5 & 10 & 15 \\
\hline Time & Volume & Volume & Volume & Volume \\
\hline 0 & 0.00000000 & 0.00000000 & 0.00000000 & 0.00000000 \\
\hline 1 & 0.00003351 & 0.00000000 & 0.00000000 & 0.00000000 \\
\hline 2 & 0.00030159 & 0.00000000 & 0.00000000 & 0.00000000 \\
\hline 3 & 0.00120637 & 0.00000000 & 0.00000000 & 0.00000000 \\
\hline 4 & 0.00335103 & 0.00000000 & 0.00000000 & 0.00000000 \\
\hline 5 & 0.00753982 & 0.00000000 & 0.00000000 & 0.00000000 \\
\hline 6 & 0.01474454 & 0.00003351 & 0.00000000 & 0.00000000 \\
\hline 7 & 0.02597050 & 0.00030159 & 0.00000000 & 0.00000000 \\
\hline 8 & 0.04222301 & 0.00120637 & 0.00000000 & 0.00000000 \\
\hline 9 & 0.06450737 & 0.00335103 & 0.00000000 & 0.00000000 \\
\hline 10 & 0.09382890 & 0.00753982 & 0.00000000 & 0.00000000 \\
\hline 11 & 0.13119291 & 0.01474454 & 0.00003351 & 0.00000000 \\
\hline 12 & 0.17760470 & 0.02597050 & 0.00030159 & 0.00000000 \\
\hline 13 & 0.23406960 & 0.04222301 & 0.00120637 & 0.00000000 \\
\hline 14 & 0.30159289 & 0.06450737 & 0.00335103 & 0.00000000 \\
\hline 15 & 0.38117991 & 0.09382890 & 39982 & 0.00000000 \\
\hline 16 & 0.47383595 & 0.13119291 & 0.01474454 & 0.00003351 \\
\hline 17 & 0.58056632 & 0.17760470 & 0.02597050 & 0.00030159 \\
\hline 18 & 0.70237634 & 0.23406960 & 0.04222301 & 0.00120637 \\
\hline 19 & 0.84027132 & 0.30159289 & 0.06450737 & 0.00335103 \\
\hline 20 & 0.99525655 & 0.38117991 & 0.09382890 & 0.00753982 \\
\hline 21 & 1.00000000 & 0.47383595 & 0.13119291 & 0.01474454 \\
\hline 22 & 1.00000000 & 0.58056632 & 0.17760470 & 0.02597050 \\
\hline 23 & 1.00000000 & 7634 & 6960 & 222301 \\
\hline 24 & 1.00000000 & 0.84027132 & 0.30159289 & 0.06450737 \\
\hline 25 & 1.00000000 & 0.99525655 & 0.38117991 & 0.09382890 \\
\hline 26 & 1.00000000 & 1.00000000 & 0.47383595 & 0.13119291 \\
\hline 27 & 1.00000000 & 1.00000000 & 0.58056632 & 0.17760470 \\
\hline 28 & 1.00000000 & 1.00000000 & 0.70237634 & 0.23406960 \\
\hline 29 & 1.00000000 & 1.00000000 & 0.84027132 & 0.30159289 \\
\hline 30 & 1.00 & $1 .($ & 55 & |7991 | - | - \\
\hline 31 & 1.00000000 & 0000 & 0000 & 0.47 \\
\hline 32 & 1.00000000 & 1.00000000 & 1.00000000 & 0.58056632 \\
\hline 33 & 1.00000000 & 1.00000000 & 1.00000000 & 0.70237634 \\
\hline 34 & 1.00000000 & 1.00000000 & 1.00000000 & 0.84027132 \\
\hline 35 & 1.00000000 & 1.00000000 & 1.00000000 & 0.99525655 \\
\hline 36 & 1.00000000 & 1.00000000 & 1.00000000 & 1.00000000 \\
\hline 37 & 1.00000000 & 1.00000000 & 1.00000000 & 1.00000000 \\
\hline 38 & 1.00000000 & 00000 & 000000 & 1.00000000 \\
\hline 39 & 1.00000000 & 1.00000000 & 1.00000000 & 1.00000000 \\
\hline 40 & 1.00000000 & 1.00000000 & 1.00000000 & 1.00000000 \\
\hline 41 & 1.00000000 & 1.00000000 & 1.00000000 & 1.00000000 \\
\hline 42 & 1.00000000 & 1.00000000 & 1.00000000 & 1.00000000 \\
\hline 43 & 1.00000000 & 1.00000000 & 1.00000000 & 1.00000000 \\
\hline 44 & 1.00000000 & 1.00000000 & 1.00000000 & 1.00000000 \\
\hline 45 & 1.00000000 & 0000 & 0000 & 0000 \\
\hline 46 & 1.00000000 & 1.00000000 & 1.00000000 & 1.00000000 \\
\hline 47 & 1.00000000 & 1.00000000 & 1.00000000 & 1.00000000 \\
\hline 48 & 1.00000000 & 1.00000000 & 1.00000000 & 1.0000000 \\
\hline 49 & 1.00000000 & 1.00000000 & 1.00000000 & 1.0000000 \\
\hline 50 & 1.00000000 & 1.00000000 & 1.00000000 & 1.00000000 \\
\hline
\end{tabular}

